\title{
LA REVISTA DIGITAL DE COMUNICACIÓN Y LOS CONTEXTOS HISTÓRICOS DE LA PUBLICACIÓN CIENTÍFICA. \\ ENCRUCIJADA A LOS DIEZ AÑOS DE LAS PRIMERAS REVISTAS IBÉRICAS \\ Y LATINOAMERICANAS DE COMUNICACIÓN EN INTERNET
}

\author{
Daniel Martí \\ Universidade de Vigo
}

\section{INTRODUCCIÓN}

Una mirada a las edades históricas de la publicación científica se propone como ejercicio reflexivo al cumplir diez años las primeras revistas académicas de comunicación publicadas en Internet desde Latinoamérica, España y Portugal. La experiencia de estas revistas científicas de comunicación, las más antiguas en la Red, transforma los modelos de edición comercial y académica de la ciencia al integrar nuevas prestaciones las aplicaciones para la edición electrónica en Internet. Al llegar las primeras revistas a los diez años no se completa ningún ciclo. Algunas anteriores en la publicación seriada impresa y otras renovadas hace pocos años como revistas digitales se encuentran en un buen momento para revisar el modo de su consolidación. Las reflexiones que siguen también esperan ofrecer perspectivas y estimular nuevas vías para bastantes revistas de comunicación que a estas alturas de 2004 todavía no han actualizado sus contenidos este año con nuevas ediciones en red.

\section{DIFERENCIAS EN LOS CONTEXTOS HISTÓRICOS}

DE LA PUBLICACIÓN CIENTÍFICA

\subsection{Escenario clásico de la comunicación científica}

Un documental en un canal televisivo vía cable comentaba que estudios paleontológicos en Europa indican una posible existencia de representaciones astronómicas anteriores en unos mil años a las más conocidas en Egipto. Como en el rastreo de la representación de unas estrellas, también en los orígenes de la comunicación científica podemos encontrar distintas similitudes y diferencias del pasado con respecto a los nuevos escenarios sobre 
tecnologías de información. El objeto de la comparación no persigue la exactitud del dato sino volver a plantear la intención, la actitud, lo que se pretendía y más o menos dónde quedó situada la cuestión de la comunicación de la ciencia y sus categorías fundamentales en sus tres épocas de cambio precedentes a la actual.

En la tradición europea occidental, solemos atribuir un origen griego a la constitución y distribución de las primeras disciplinas científicas con un objeto, métodos y autores reconocidos en su investigación. Parece que Atenas en el siglo de Pericles, v a. J.C., alcanzó niveles económicos y políticos con una estabilidad que facilitó la separación de Mito y Filosofía. Tras la demarcación filosófica se formularon las primeras especialidades científicas, por los mismos Sócrates, Platón, Aristóteles -y tantos otros-, que consignaron los conocimientos de nuestro medio y entorno y que organizaron en un conjunto integrado sus exposiciones y muchas otras heredadas o recibidas de autores anteriores.

Los imaginarios de un universo ordenado presentan contradicciones con teorías científicas contemporáneas, pero proyectan un paradigma lógico y jerárquico de división y organización del saber todavía vigente en otras teorías actuales de la información, del conocimiento, de la divulgación y de las demás actividades intelectuales implicadas en la comunicación de la ciencia.

Junto con las primeras clasificaciones y distribuciones de disciplinas, en la antigüedad ateniense el discurso científico también configuró unas formas retóricas más adecuadas para la discusión, la transmisión y la recopilación según los sistemas de producción, distribución y conservación de documentos.

Tratados, manuales, disertaciones, diálogos... colaboraron en la primera construcción de los públicos interesados en el aprendizaje y la comunicación científicas.

\subsection{Impronta universitaria de la comunicación científica}

El cambio a un nuevo paradigma se anticipa en el contexto de un diferente escenario religioso y político que, en la Baja Edad Media, construye centros civiles y religiosos donde se traducen, comentan y archivan textos anteriores y contemporáneos. 
Constituirán las bases de la primera formación e investigación en universidades. También son una refundación, de mayor difusión social y más institucional, de las publicaciones académicas de culturas antiguas.

Como materiales para esa formación en centros específicos aparecen las publicaciones recopilatorias y monográficas, que desarrollan los géneros académicos en nuevas variantes, precedentes de posteriores publicaciones monográficas o seriadas.

Sin embargo, en los siglos de la comunicación científica vinculada a la universidad, se perciben signos de fragmentación del escenario sapiencial antiguo. Se perciben horizontes diversos tanto en los planteamientos teóricos y metodológicos de la investigación como en la misma definición y clasificación de las ciencias y las artes.

Entre otras tendencias, se acentúa una especialización progresiva y una contextualización cultural de la investigación científica al lento ritmo de la constitución en estados de algunas de las regiones nacionales en Europa y América.

\subsection{Publicaciones científicas impresas}

Sin demérito de la tecnología y del arte del papel y de la tinta, los sistemas de reproducción e impresión catalizan la evolución cultural y nacional de siglos siguientes. Como se ha recordado en los últimos años (Guédon, 2001), coincide además con el primigenio enfrentamiento democrático a las monarquías teocráticas, de economía colonial, que siglo tras siglo ven debilitar su hegemonía sobre otras naciones europeas.

El esbozo contextual en torno a Oldenburg permite intuir la influencia de los valores sociales cifrados en el individuo y la propiedad sobre otras dimensiones culturales y en la investigación y la comunicación científicas. A pesar del escueto marco, se vislumbran algunas explicaciones del nacimiento en Inglaterra y en Francia de las primeras revistas de sociedades científicas.

Incluso se han apuntado dos estrategias editoriales, todavía no directamente competitivas, que se debatieron como primer modelo de la gestión de la información científica: 
1. política de autentificación y reconocimiento del valor de la propiedad intelectual de los investigadores, que parece señalar el proyecto inglés, $\mathrm{y}$

2. planteamiento editorial francés también orientado a los cambios y evolución de la actualidad de la ciencia.

En la sucesión de hegemonías nacionales y en el modelo de comunicación científica se impusieron los británicos.

Desde el punto de vista del contexto de los contenidos, el macrotema de la división del saber ha pasado a convertirse en un diálogo bibliográfico en torno a los principios de la ciencia y los discursos sobre métodos. Estos precedentes y primeros ensayos de la publicación seriada no sustituyen las ediciones escolares de los tratados y manuales o el género epistolar, pero también tienen consecuencias retóricas y formales en ellos.

Además de la aportación de las revistas a la división y especialización de las ciencias (y otros efectos acumulables a objetivos de entornos y paradigmas anteriores), el desarrollo de las revistas salta las fronteras de la comunidad de investigadores y de las comunidades de formación, hasta penetrar en intereses particulares de la esfera civil. La comunicación científica y sus impresiones se han extendido desde el siglo XVII a los eventos sociales de divulgación (Panza y Presas, 2002), como ciclos de lecturas y conferencias o las colecciones, exposiciones y museos..., ampliando expectativas sociales en torno a nuevas disciplinas y enseñanzas en la universidad e institutos de investigación.

Era necesario que la reproducción y la difusión social de textos se encauzara con la experiencia normalizadora de la edición monográfica y ad hoc. En las revistas la normalización es nacional y, en general, compleja; casi imposible todavía en algunos países para otros tipos de publicaciones seriadas como las residentes en Internet. Tampoco la normalización en la literatura gris y de corta circulación (Rubio, 2002) se ha asentado, por razones similares a la anterior. Así pueden verse todavía revistas, en los dos soportes, sin normalizar tanto en Portugal o España como en Latinoamérica. 


\section{LA REVISTA EN LA COMUNICACIÓN CIENTÍFICA}

\subsection{Funciones de la revista científica}

Enfocando la revista científica, desde el dinámico y tecnológico presente, selecciono listas de funciones y de inconvenientes de la edición científica seriada que me parece imposible que se formularan en esta forma de no existir las revistas digitales y otras aplicaciones informáticas y formatos de edición en línea como los que usamos diariamente.

John W. T. Smith (1999) distingue dos tipos de funciones, explícitas y menos notorias, en el papel desempeñado por las revistas científicas.

Seis primeras funciones, explícitas, más comentadas (Smith 1999, 81), se pueden resumir como:

- selección editorial de los materiales en función de la orientación temática en el estilo de la revista y/o según el posible interés para sus lectores,

- control de la calidad de los contenidos recibidos por el equipo editorial o los revisores que colaboran en la edición,

- control de la calidad de redacción y representación en los textos e imágenes a cargo de los responsables de la producción editorial,

- atribución de reconocimiento al trabajo de los investigadores por la publicación,

- consecución de reputación ante los lectores o bibliotecarios para las mismas cabeceras científicas merced a su autoridad, rigor, antigüedad, etc.

- difusión o divulgación de información científica.

Aunque las funciones ocultas o subyacentes han recibido menor atención, las revistas científicas también han sido agentes activos en:

- definición de las áreas de cada disciplina, a través de los editoriales, de selección y de las revisiones de documentos,

- definición de la comunidad de los lectores. Función más evidente en el caso de las nuevas disciplinas o de las áreas emergentes, 
- archivo documental de la especialidad, indirectamente, a través del archivo documental en bibliotecas y centros de recursos (Smith 1999: 81-82).

\subsection{Inconvenientes de la revista científica impresa}

Un caro y lento proceso de producción impresa disminuye algunos de los valores presumibles en la publicación científica seriada. Tanto funciones explícitas de las revistas, como la construcción de las áreas disciplinares, de los públicos y de unas bases documentales colectivas, han resultado condicionadas por la falta de visibilidad de sus contenidos y por la presión jerárquica de las autoridades y los criterios comerciales en la gestión editorial.

En opinión de expertos en economía de la comunicación científica, por el tipo de industria y de producción de textos impresos. Para Weary y Schutz (1997, sobre revistas de física), las revistas impresas de información científica:

- retrasan la difusión de conocimientos científicos,

- fijan una «agenda de contenidos» y secciones en estructuras poco flexibles $y$,

- solidifican o canonizan las versiones publicadas de los documentos, como textos definitivos.

Desde la perspectiva del uso y de la satisfacción de los objetivos esperados por lectores, coleccionistas o bibliotecarios, la comunicación científica también presenta distintos inconvenientes, entre los que destacaré la falta de visibilidad del texto científico y la lucha por la reputación.

Sin despreciar las técnicas de observación, fenomenológicas o sociológicas, imprescindibles para la cuantificación y el análisis, deseo mencionar aquí el interés de los datos de lectura o de uso de la información científica, en disciplinas auxiliares como la informetría (Spinak 1996, Morales 1995 y Morales 1997 citado en Araujo y Arencibia 2002). Desarrollada en Centroeuropa desde mediados de la década de los años ochenta, a partir de la bibliometría y de la cienciometría, podemos encontrar técnicas de cuantificación de producción y de uso de información, a partir de estu- 
dios más clásicos de productividad de autores (Lotka 1926), de reconomiento y dispersión de fuentes investigadoras (Bradford 1934) u otros aspectos estadísticos de los contenidos (Zipt 1949).

Entre los estudios de usos de la información registrada y el análisis infométrico de citas se pueden encontrar textos científicos sin citación exterior alguna (10\% en medicina o el $7 \%$ prehistoria, Terrada et al. 1992). Otros documentos, en los últimos diez años, no han sido citados una sola vez. Sobre una amplia muestra, Lesk (1997) encuentra un 22\% de los artículos y revisiones de ciencias nunca citados; el 48\% de los textos de ciencias sociales y jel 93\% de los textos humanísticos! sin que nadie les hiciera referencia entre 1984 y 1993. Las ciencias humanas y sociales tampoco figuraban igualmente representadas, se medía con parámetros inadecuados y había otros problemas, conocidos y discutidos, de los índices y de los análisis de citas.

Tampoco en lo que se refiere a la reputación y el reconocimiento de autoridades las revistas impresas han supuesto un cauce inmejorable para la expresión y la comunicación de la calidad científica.

El asunto del reconocimiento, con diferencias tan importantes entre unas ciencias y otras, es fuente de discusiones en la que tienen intereses los estados, las grandes industrias, incluidas las culturales, y el orgullo humano desde sus variados laboratorios y departamentos.

La misma necesidad ha ido desarrollando una cienciometría (Brookes 1990), a partir de la bibliometría europea de la década de los años setenta. Pretende prestar sus servicios a la sociología de la ciencia y al establecimiento de políticas estatales de investigación (Araujo y Arencibia, 2002). Cuantifica la producción por disciplinas, la productividad y creatividad de los investigadores y de las estructuras de su difusión. Como en el caso de la informetría, el análisis de la comunicación científica también demanda precisiones culturales y políticas regionales que la desplazan en las frecuentes reclamaciones a las listas científicas nacionales y en los conflictos de intereses (como factores de impacto o específicamente como conflictos de intereses con la industria; Camí 1995). 
A partir de las primeras leyes como la de Bradford 1934 (suscitada para racionalizar el gasto bilbiográfico de la investigación después de la gran depresión) y la ley de Lotka de la productividad científica, se ha centrado la calidad y el valor de la investigación científica en torno a revistas nucleares de las disciplinas evaluadas con el precedente del Institute of Scientific Information (ISI) en el Science Citation Index.

La identificación de las revistas nucleares con «lo más granado» de la información científica o incluso como el núcleo de una ciencia resulta impropia. Sin embargo, debido a la función prescriptora o de gatekeepers ejercida por los editores de esas cabeceras, se produce tal identificación, como se puede comprobar en decisiones sobre políticas científicas nacionales y en la opinión pública de los lectores sobre el estado de la ciencia (Guédon, 2001). Aun cuando parezca obvio que la excelencia o calidad reconocida en el sistema jerárquico dirigido por los editores no garantiza la calidad y excelencia científicas en sí mismas consideradas.

Reconociendo la conveniencia de una función editorial especializada y las ventajas de una revisión por pares de los documentos que se han de seleccionar por consejos editoriales para su publicación, desde mediados de los setenta, los derechos de autor y la reputación atribuida han multiplicado el valor de mercado de la publicación científica ante bibliotecas, universidades, institutos y alumnos/lectores. Como en otros mercados cada vez más globales, se ha producido una concentración de distribuidores y de títulos nucleares de las disciplinas, acompañada de un importante encarecimiento de los productos científicos publicados, por motivos de negocio y de la concentración de la producción y la distribución para un mercado desarrollado y en expansión (p. ej.: ARDE, 2004).

\section{REVISTAS DIGITALES DE COMUNICACIÓN Y SEDES} WEB

\subsection{Las primeras revistas digitales sobre comunicación}

Investigaciones científicas han sido objeto de publicación seriada disponible en world wide web desde los años 90. Éxitos 
editoriales, en el sector de la información y de las revistas, empujaron muchos otros proyectos siguiendo la estela de Wired (1993), contestación al y desde el mundo académico, bajo la impronta de Nicolas Negroponte, nacida cerca del MIT (Instituto Tecnológico de Massachusetts) o de First Monday (1996). Con el desarrollo de la publicación electrónica, las revistas académicas han pasado a compartir formato digital con la edición impresa o como proyectos diseñados específicamente para distribución por correo electrónico o en Internet.

Una de las citas habituales en el mundo hispanohablante define la revista académica digital como «conjunto de artículos ordenados, formalizados y publicados bajo la responsabilidad de una institución científica o técnica a través de redes teleinformáticas» (Barrueco, García Testal y Gimeno, 1996). En otras palabras, no se percibe marca entitativa salvo las relativas al soporte. Después, Villalón y Aguillo (1998, en Baró y Ontalba, 2002) detallan las categorías «artículo científico» como texto completo, resúmenes o simples sumarios de contenidos y la «responsabilidad de la institución científica o técnica» con respecto a la autenticidad y la calidad de la información publicada.

Tras diferentes formas de traslado de ediciones impresas a Internet, las distribuidoras comerciales de información científica han instaurado también sus propias sedes electrónicas para la transacción de distintos formatos electrónicos por venta o suscripción. Por las condiciones de difusión electrónica, las revistas digitales igualan o superan la difusión científica americana (Mercer 2000). También crece y aumenta el impacto de las hispano-portuguesas (Aliaga 2002). En opinión de Solomon (2002), la liberación de los contenidos para su difusión a través de la red está en manos de los académicos. Las revistas no tienen por qué perder su ascendiente sobre la acreditación y los derechos, sin embargo pueden flexibilizar sus políticas de edición y sus contratos de derechos.

Desde Smith (1999) y otros (Rodríguez 1999, Aliaga 2002, Baró y Ontalba 2002) se distinguen dos niveles iniciales de digitalización: 
1. el traslado de la política y los formatos de ediciones impresas anteriores o simultáneas, con respecto a

2. los proyectos editoriales exclusivos para la red.

Nuevas ediciones electrónicas se añadieron al papel en la información científica de comunicación en español y portugués entre 1994 y 1998. Revistas digitales como Comunicación y Sociedad (www.unav.es/cys/), Telos (www.campusred.net/telos/), Pixel Bit (www.sav.us.es/pixelbit/), Comunicar (www2.uhu.es/comunicar /revista.htm), Pensamiento Científico (www.ciencytec.com/pc/), Quark (www.imim.es/quark/), Relieve (www.uv.es/RELIEVE/), Razón y Palabra (www.cem.itesm.mx/dacs/publicaciones/logos/), Revista CIMJ Centro de Investigação Media e Jornalismo (www.cimj.org/ publicacoes.asp?tipub=Revista), Latina (www.ull.es/publicaciones/latina.htm), Ámbitos (www.ull.es/publicaciones/latina/ambitos/ ambitos.htm), Chasqui (www.comunica.org/chasqui/), Ciberlegenda (www.uff.br/mestcii/rep.htm) o Hipertextos (http://hiper-textos. mty.itesm.mx/index.htm), ahora como Global Media Journal.

Algunas mantienen la maqueta y la estructura de contenidos de la versión en papel; otras simplemente han imitado en la red formatos bastantes estandarizados de las ediciones web. A pesar de la pobre versión electrónica que presentan algunas de estas pioneras, la coedición electrónica bajo la misma imagen y cabecera podría ofrecer servicios añadidos a bajo coste, naturales en el entorno web en el que se sitúan. Por ejemplo (Baró y Ontalbo, 2002): búsqueda interna, actualización de secciones y artículos, recursos seleccionados y comentados, archivos complementarios que incluyan tablas o gráficos de datos y en varios formatos, líneas de debate y discusión, etc.

Estas oportunidades tecnológicas no están siendo utilizadas por la mayoría de las revistas de ciencias sociales en lenguas hispanas. Simplemente han aplicado modelos de edición impresa inútiles para una gestión tecnológica de los contenidos. Lo que los usuarios consideramos como defectos en muchas revistas de esta generación no sólo se debe a una deficiente estrategia editorial justamente criticada por Loría y Magaña (2001). 
También se ha debido a la fase de introducción de Internet en la que ha podido destacar la arquitectura de información propia de portales de contenidos como modelo dominante de sitio o sede web con información. Este hipertexto de múltiples documentos enlazados a distintos niveles con su domicilio o URL principal (aludo a la definición del World Wide Web Wonsortim de website y homepage) asienta la imagen de un espacio nuclear estático bien diferente de la experiencia de uso y de las distintas modalidades de navegación que pueden ofrecer las sedes web.

Para las revistas, una etapa de digitalización más profunda exige diferenciar los interlocutores y los usos normalizados y acotados en las últimas décadas de las publicaciones impresas según la experiencia de los usuarios de Internet. El error de la asociación a una arquitectura y sus modelos de desarrollo para sedes condiciona las demás modalidades de interacción y puede derivar hacia otros formatos y estructuras como ha ocurrido con la edición en pre-print habitual en astronomía o física (wikipedia: electrónic publishing). La publicación en colaboración, de acceso público en formato wiki o weblog, ya cubre servicios potenciales de revisión de actualidad y oferta de servicios nocionales y enciclopédicos. El problema de la migración de públicos no sólo afecta a la arquitectura, el estilo y la política editoriales de revistas, sino que se extiende por millones de páginas de empresas, de organizaciones y, lo que es más grave, a la mayor parte de los servicios públicos de los que se informa en red.

4.2 Servicios web de información científica sobre comunicación

La coordinación científica, la integración en proyectos empresariales y sociales o la divulgación desde los más pequeños a los mayores marcan las exigencias de esta época con la experiencia de las anteriores. Las revistas ocupan un espacio privilegiado para atender a las demandes de fondo. Pero no son las únicas estructuras posibles en Internet y tendrán que decidir sus estrategias. A la vista de los proyectos digitales originales sobre comunicación, parece oportuno considerar cambios en el modelo de 
revista tomando en consideración desde las primeras sugerencias sobre la importancia de los servicios de valor informativo añadido y la apertura a la participación de los usuarios (Okerson, 1997; Hunter, 1997; Smith 1999). Una experiencia de este tipo de propuestas puede navegarse en el movimiento por el libre acceso a los recursos (Open Archives Iniciative) o las bibliotecas virtuales.

Las reformas de modelo y los ejemplos no culminan el desarrollo de la interacción. Ya se ensayan formas de interacción con el texto, el autor, con el editor (Aliaga y Suárez, 2002: 1). Por ejemplo, son interacciones con los contenidos los hilos de una lista de discusión o las secuencias de comentarios que ha suscitado; la comunicación y actualización de los datos originales, sucesivos, tablas, gráficos...

Aplicaciones informáticas relacionadas con los motores de búsqueda y las bases de documentos están depurando la recuperación automática de información preliminar para la acreditación de autores o de los enlaces (Arms 2002). Las mismas y otras prestaciones de la búsqueda se están usando además en el rediseño y configuración de las páginas de presentación o de las bases de datos en las sedes. No sólo se facilita la cuantificación, sino que sirven a un rediseño o una reconfiguración más sencilla de la arquitectura, en función de los intereses dominantes en una comunidad científica o de los usos más frecuentes de la información en un entorno. Una realidad que sin cerrarse en la automatización ofrece parte de las ventajas «democráticas» que Nadasdy (1997) o Smith (1999) vislumbraban en su modelo de revistas electrónicas deconstruidas.

Para la gestión editorial son relevantes las estadísticas instantáneas de uso, la recuperación de enlaces o avisos automáticos recibidos, las votaciones y sugerencias de los lectores. La riqueza de contenidos y de operaciones en la navegación dispone de un abanico paralelo de formatos de edición, archivo e intercambio. Además se trabaja en nuevas versiones de sistemas operativos, que faciliten una navegación multimedia entre los distintos tipos de archivo (tanto en el creciente software libre, como por parte del Windows Longhorn, que se esperaba para 2005 y que anuncia un mayor retraso). 
Con prestaciones fáciles de instalar y de gestionar, se desdibujan las fronteras de la edición académica clásica detrás de unas políticas de visibilidad ante los buscadores o la multiplicación de navegantes con tipos complementarios de uso. La notoriedad pública se relaciona con la pérdida de reputación científica, aunque tampoco es ninguna ley ni axioma.

La aproximación entre el artículo científico y el informe profesional u otras formas de literatura gris supone una política editorial experimentada en Internet y no tiene por qué perjudicar el valor científico del resto de los contenidos. Información interesante al servicio de los profesionales se encuentra en sus asociaciones, con publicaciones seriadas, como Etcétera, Pulso del periodismo, Capçalera o Sala de Prensa, aunque también haya sido asunto en revistas académicas como Estudios del Mensaje Periodístico o en monografías de la Sociedad Española de Periodística.

No quiero olvidarme de los espacios y formatos web relacionados y en ocasiones complementarios por la interacción de la tecnología sobre la información científica de comunicación y que ocasionalmente puede interesar y afectar como ocurre con E-ciencia, Wired News, el canal de tecnología de Terra, Diario Red, o en noticias y análisis en weblogs o boletines (sin ISSN ni publicidad) como Error 500, Comunisfera o Infovis.

El incremento y los nuevos tipos de público y de interacciones complican la evaluación de la calidad científica, para la que ahora contamos con herramientas de mayor capacidad y muy variadas prestaciones. En la actualidad se sigue desarrollando la metainformación documental y su gestión por aplicaciones informáticas para prestar servicios del tipo de los agentes de certificaciones en el contexto inmediato de enlaces acreditados.

En definitiva, no parece perjudicial este tipo de esfuerzo por recuperar la capacidad de comunicación civil de las instituciones científicas, y en concreto de la universidad, con su entorno empresarial y social en general, y/o reforzar la formación continuada y la categoría científica de los contenidos servidos por la asociaciones profesionales y las corporaciones. 
Para no olvidar del todo los objetivos sapienciales de la congruencia entre investigaciones científicos, para el diálogo entre conclusiones de distintas perspectivas, se están ofreciendo servicios específicos, ya no desde revistas sino sobre todo desde portales de comunicación y observatorios de medios de comunicación.

En la corta selección siguiente se pueden ver servicios tradicionalmente atribuidos a revistas científicas de comunicación: On Line Journalism, Cyberjournalist, Ciberperiodismo de Press.Net, Infoamérica, Incom o en Innovatec-Innovarium. Algunos incluso publican newsletters o boletínes semanales o mensuales como CiencyTec, Alaic, Obercom pt, Observatec y otros similares, que se podrían considerar en alguna medida competidores con respecto a las ediciones de publicaciones clásicas.

Portales y observatorios, en general, se componen de una arquitectura de servicios similar y consistente, con agendas o información de eventos, boletines o enlaces a sus publicaciones o bibliografías básicas de la especialidad, directorios o guías profesionales clasificadas por países y/o por sectores de medios, zonas para grupos de trabajo o de proyectos, glosario y otros recursos teóricos, académicos o docentes. En este momento, todavía son excepción los que trabajan con metainformación documental o rankings y estadísticas públicas de usos. Como algunas sedes ya han dado el paso, es previsible que a medio plazo se extienda o incluso se estandarice entre sedes de acceso público a información científica de comunicación.

Para seguir la evolución de este capítulo español, portugués y latinoamericano de publicaciones digitales seriadas sobre comunicación contamos con algunos servicios actualizados en portales de comunicación y bibliotecas universitarias o de centros de investigación. Con distintos criterios de cobertura editorial o de selección para usos educativos e investigadores, estas listas de revistas de comunicación (incluyendo alguna breve descripción, con algunos fallos de enlace y enlaces a revistas que no se han seguido editando) se pueden encontrar en Scielo.org, Universia, portal universitario, Biblioteca Complutense, Infoamérica, Asociación 
Latinoamericana de Investigadores de la Comunicación (ALAIC), relaciones por área y países elaboradas por Daniel E. Jones en la revista Zer 6-11, 14, 15 de 1999 a 2003 o junto a otras fuentes documentalistas (Codina 1998), pedagógicas (web personal de Francisco Aliaga) o de otras disciplinas que incluyen textos relacionados con la comunicología. 


\section{REFERENCIAS BIBLIOGRÁFICAS}

Aliaga, F. y SuÁrez Rodríguez, J. (2002) «Tendencias actuales en la edición de revistas electrónicas: nueva etapa en RELIEVE», Revista Electrónica de Investigación y Evaluación Educativa, vol. 8, n. ${ }^{\circ} 1$.

Aguado, E. et al. (2004) «Red Alyc: la hemeroteca en línea en ciencias sociales», Ciencia: ergo sum, 11, 1.

Araujo, J. A. y Arencibia, R. (2002) «Informetría, bibliometría y cienciometría: aspectos teórico-prácticos», Acimed, vol. 10, n. ${ }^{\circ} 4$, julio-agosto 2002.

Polo, A. «Estudio Estadístico sobre las Revistas Digitales en España», en noticias 15 mayo 2004. http://www.ardeweb.org/not_040604.php.

ARms, W. Y. (2002) «WHAT ARE THE ALTERNATIVES TO PEER REVIEW? Quality Control in Scholarly Publishing on the Web», The Journal of Electronic Publishing, 8, 1.

Baró, J. y Ontalba, J. A. (2002) «Las revistas digitales académicas españolas de documentación: análisis de las existentes y propuesta de modelo», en http://www.uoc.edu/web/esp/articles/revistas_digitales.html, 19 febrero 2002.

Barrueco, J. M.; García Testal, C. y Gimeno, M. J. (1996) «Una aproximación a las revistas científicas en formato electrónico», Revista Española de Documentación Científica, vol. 19, n. ${ }^{\circ}$ 3, p. 304-313.

BRADFORD, S. C. (1934) «Sources of information on specific subject», Engineering, 137, 85-86. Citado por Araujo y Arencibia, 2002.

BROOKES, B. C. (1990) «Biblio-sciento-informetrics? What are we talking about?», Informetrics. Amsterdam, Elsevier, p. 31-43. Citado por Araujo y Arencibia, 2002. 
Camí, J. (1995) «Investigación científica y conflicto de intereses», Quark 1.

GuÉDON J. C. (2001) In Oldenburg's Long Shadow: Librarians, Research Scientists, Publishers, and the Control of Scientific Publishing. Association of Research Libraries Publications.

Lesk, M. (1997) Books, Bytes and Bucks: Practical Digital Libraries, TBA, San Francisco.

Loría y Magaña (2002) «Revistas académicas y el Conacyt», La Jornada.

http://www.jornada.unam.mx/2002/jun02/020603/cienloria.html.

LotKA, A. J. (1926) «The frequency distribution of scientific productivity», J. Washington Acad. Sci. 16 (12), 317-323. Citado por Araujo y Arencibia, 2002.

Mercer L. S. (2000) «Measuring the use and value of electronic Journals and Books», Issues in Science and Technology Librarianship, n. ${ }^{\circ} 25$.

Morales, M. (1995) «La bibliotecología, la cienciología y la ciencia de la información y sus disciplinas instrumentales: su alcance conceptual», Revista de Ciencias de la Información. Segunda Época. Facultad de Ciencias de la Información, Universidad Complutense de Madrid. 26(2), 70-88. Citado por Araujo y Arencibia, 2002.

Morales, M. (1997) «La informetría y las fuentes de información personales e institucionales: su importancia en relación con la información de inteligencia», Cienc. Inform. 28 (2), 207-217. Citado por Araujo y Arencibia, 2002.

Okerson, A. (1997) «Recent Trend in Scholarly Electronic Publishing», en Seminar on Multimedia Scholarly Publishing, Helsinki, May 29. 
Panza, M. y Presas, A. (2002) «La divulgación de la ciencia en el siglo XIX: la obra de Flammarion», Quark, 26, octubre - diciembre 2002.

Rodríguez, G. (1999) «Revistas electrónicas: cibernautas y/o papirófilos. Cambios en la comunicación científica», Revistas de Investigación Educativa, vol. 17, n. ${ }^{\circ}$ 2, p. 491-494. Citado por Araujo y Arencibia, 2002.

Rubio Liniers, M. C. (2002) «Bibliometría y Ciencias Sociales», CLIO, n. ${ }^{\circ} 7$.

Smith, J. W. T. (1999). «The Deconstructed Journal -a new model for Academic Publishing», Learned Publishing, vol. 12, n. ${ }^{\circ} 2$, Abril 1999.

Solomon, D. J. (1999) «Is it Time to take the paper out of Serial Publication?», Medical Education On Line, Michigan State University, 4, 7 .

Solomon, D. J. (2002) «Talking past each other: Making sense of the debate over electronic publication», First Monday, 7, 8.

SPINAK, E. (1996) Diccionario Enciclopédico de Bibliometría, Cienciometría e Informetría, Caracas, UNESCO, p. 34-131. Citado por Araujo y Arencibia, 2002.

Terrada, M. T. et al. (1992) Índice de citas e indicadores bibliométricos de las revistas españolas de medicina interna y sus especialidades, Barcelona, Instituto de Estudios Documentales e Históricos sobre la Ciencia.

Villalón, J. y Aguillo, I. F. (1998) «Revistas electrónicas en ciencias sociales y humanidades», Revista Española de Documentación Científica, vol. 21, n. ${ }^{\circ}$ 3, p. 303-316.

WheAry, J. y Schutz, B. F. (1997) «Living Reviews in Relativity: Making an electronic journal live», Journal of Electronic Publishing, septiembre 1997, 3, 1.

ZIPT, G. K. (1949) Human behavior and the principle of least effort, Addison Wesley. Citado por Araujo y Arencibia, 2002. 